\title{
Incidental Diagnosis of Pediatric Arytenoid Cartilage Dislocation During Videofluoroscopic Swallowing Study: A Case Report
}

\author{
Yonghyun Lee, $\mathrm{MD}^{1}$, Hankyul Park, $\mathrm{MD}^{2}$, Jae Eun Park, MD${ }^{1}$, Seung Ki Kim, MD¹, \\ Eun Sook Park, $\mathrm{MD}^{1}$, Dong-wook Rha, MD, $\mathrm{PhD}^{1}$ \\ ${ }^{1}$ Department of Rehabilitation Medicine and Research Institute of Rehabilitation Medicine, \\ Yonsei University College of Medicine, Seoul; \\ ${ }^{2}$ Department of Rehabilitation Medicine, Seoul Rehabilitation Hospital, Seoul, Korea
}

\begin{abstract}
Arytenoid cartilage dislocation is one of the most common mechanical causes of vocal fold immobility. The most common etiologies are intubation and external trauma, but its incidence is lower than $0.1 \%$. Its symptoms include dysphonia, vocal fatigue, loss of vocal control, breathiness, odynophagia, dysphagia, dyspnea, and cough. Although there are some reports of arytenoid cartilage dislocation in adults, there are only few reports on its occurrence in children. It is particularly difficult to detect the symptoms of arytenoid cartilage dislocation in uncooperative pediatric patients with brain lesions without verbal output or voluntary expression. We report a case of arytenoid cartilage dislocation with incidental findings in a videofluoroscopic swallowing study performed to evaluate the swallowing function.
\end{abstract}

Keywords Arytenoid cartilage, Fluoroscopy, Hypoxic ischemic encephalopathy

\section{INTRODUCTION}

Arytenoid cartilage is a paired, pyramid-shaped cartilage in the larynx, which plays an important role in sound production by participating in the movements of the vocal fold. The arytenoid cartilage can be dislocated due to several reasons, the most common being trauma from tracheal intubation (77.8\%), and second is external trauma (17.4\%) [1]. The incidence of arytenoid cartilage dislocation after tracheal intubation was reported as $0.023 \%-0.1 \%$ in previous studies on adults $[2,3]$; however, much less in children. The most common symptom is hoarseness [2]. In addition, dysphagia, sore throat, and stridor may occur. Accurate diagnosis of arytenoid cartilage dislocation is important because it is a curable cause of hoarseness associated with intubation and laryngeal

Received April 15, 2019; Accepted June 19, 2019

Corresponding author: Dong-wook Rha

Department of Rehabilitation Medicine and Research Institute of Rehabilitation Medicine, Yonsei University College of Medicine, 50-1 Yonsei-ro Seodaemun-gu, Seoul 03722, Korea. Tel: +82-2-2228-3717, Fax: +82-2-363-2795, E-mail: medicus@yonsei.ac.kr

ORCID: Yonghyun Lee (https://orcid.org/0000-0002-0997-3672); Hankyul Park (https://orcid.org/0000-0002-6695-9156); Jae Eun Park (https://orcid. org/0000-0001-8790-7681); Seung Ki Kim (https://orcid.org/0000-0002-8823-6258); Eun Sook Park (https://orcid.org/0000-0002-9144-3063); Dongwook Rha (https://orcid.org/0000-0002-7153-4937).

(c) This is an open-access article distributed under the terms of the Creative Commons Attribution Non-Commercial License (http://creativecommons.org/ licenses/by-nc/4.0) which permits unrestricted noncommercial use, distribution, and reproduction in any medium, provided the original work is properly cited. Copyright ( 2020 by Korean Academy of Rehabilitation Medicine 
trauma. Any change in voice and scarring or bruising on the neck can be suspicious signs. Direct visualization with laryngoscopy can confirm the diagnosis. To detect the exact etiology, additional studies can be utilized. Laryngeal electromyography can help distinguish between neurological cause and structural abnormalities by analyzing muscle recruitment $[4,5]$. A neck computed tomography (CT) scan can be used to distinguish nerve paralysis and identify structural damage such as thyroid cartilage fractures [6]. Arytenoid cartilage dislocation has been reported in adults; however, it is rarely found in children. In particular, it is more difficult to diagnose arytenoid cartilage dislocation in patients with brain damage who do not vocalize spontaneously. Arytenoid dislocation can affect the swallowing function, and these findings are easily missed on the videofluoroscopic swallowing study (VFSS). We report a case of arytenoid cartilage dislocation incidentally diagnosed during VFSS that was performed for the evaluation of dysphagia secondary to hypoxic-ischemic encephalopathy.

\section{CASE REPORT}

The patient was a 6-year-old girl who became quadriplegic due to hypoxic-ischemic encephalopathy following cardiac arrest. She was born at the gestational age of 40 weeks with a birth weight of $3.4 \mathrm{~kg}$, and developed normally. On December 23, 2016, sudden cardiac arrest occurred during recovery after strabismus surgery in the ophthalmology department. After 10 minutes of cardiopulmonary resuscitation including endotracheal intubation, the return of spontaneous circulation was identified. Diffusion-weighted MRI images taken 2 weeks after the event showed increased signal intensity in the bilateral occipital lobes, thalamus, and basal ganglia (Fig. 1A). A T2-weighted FLAIR (fluid-attenuated inversion recovery) image taken 3 months after the event showed diffused cerebral atrophy of the thalamus and basal ganglia, and encephalomalacia of bilateral occipital lobes (Fig. 1B). On admission to our rehabilitation hospital on May 25,2017 , she did not show any spontaneous vocalization with a Coma Recovery Scale score of 4 points. Spasticity was measured as grade 2 on the Modified Ashworth Scale for both upper and lower extremities. Bilateral hyperreflexic deep tendon reflexes of all four extremities were observed with Babinski's sign and ankle clonus. Auscultation of the chest revealed stridor in the supine position; however, not in the lateral decubitus position. A neck CT taken to evaluate the upper airway only showed diffuse soft tissue swelling and suspicious airway stenosis. Since she was on nasogastric tube feeding, a VFSS was performed to evaluate her swallowing function. During the VFSS, anterior collapse of posterior vocal cords was incidentally observed (Fig. 2A, 2B). This collapsing movement was associated with inspiratory stridor. Using video laryngoscopy, anterior dislocation of arytenoid cartilage obstructing the airway during inspiration was confirmed (Fig. 3). After a month of observation, stridor during inspiration gradually improved and anterior dislocation of the arytenoid cartilage have been alleviated on repeat
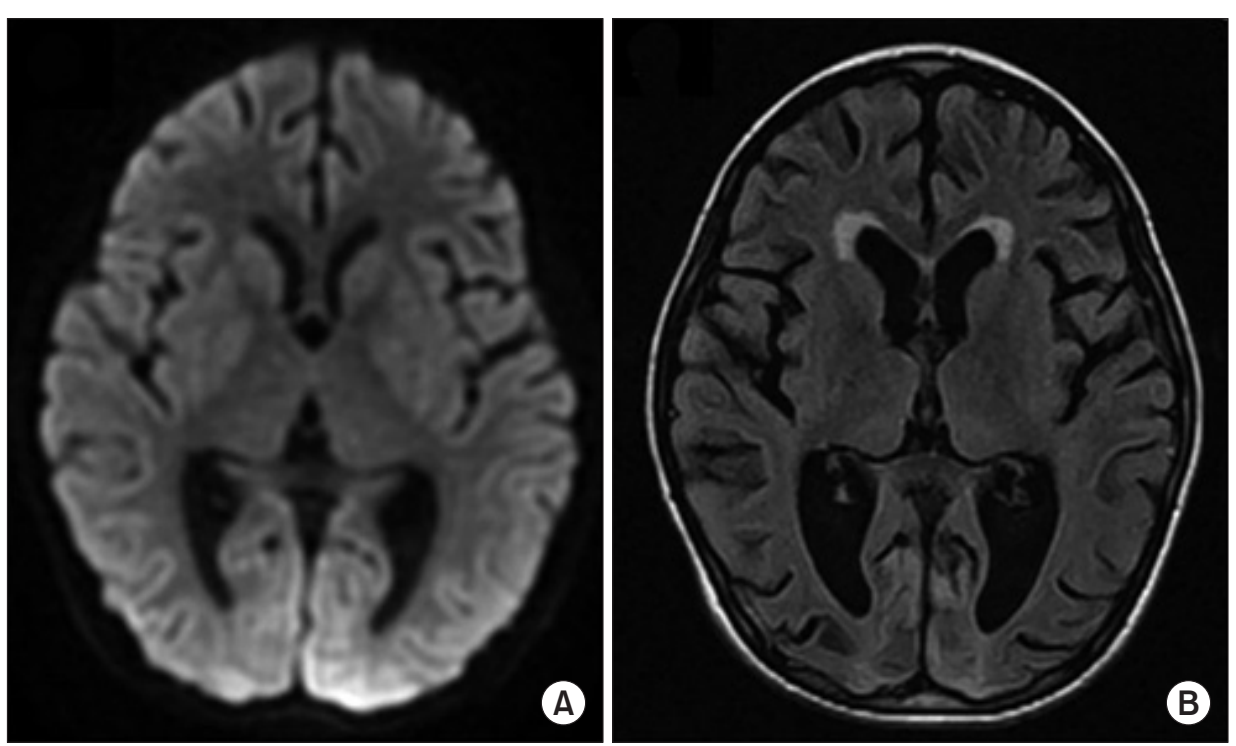

Fig. 1. A diffusion-weighted magnetic resonance imaging images taken 2 weeks after the event showed increased signal intensity in bilateral occipital lobes, thalamus and basal ganglia (A). A T2weighted FLAIR image taken 3 months after the event showed diffused cerebral atrophy of thalamus and basal ganglia, and encephalomalacia of bilateral occipital lobes (B). 

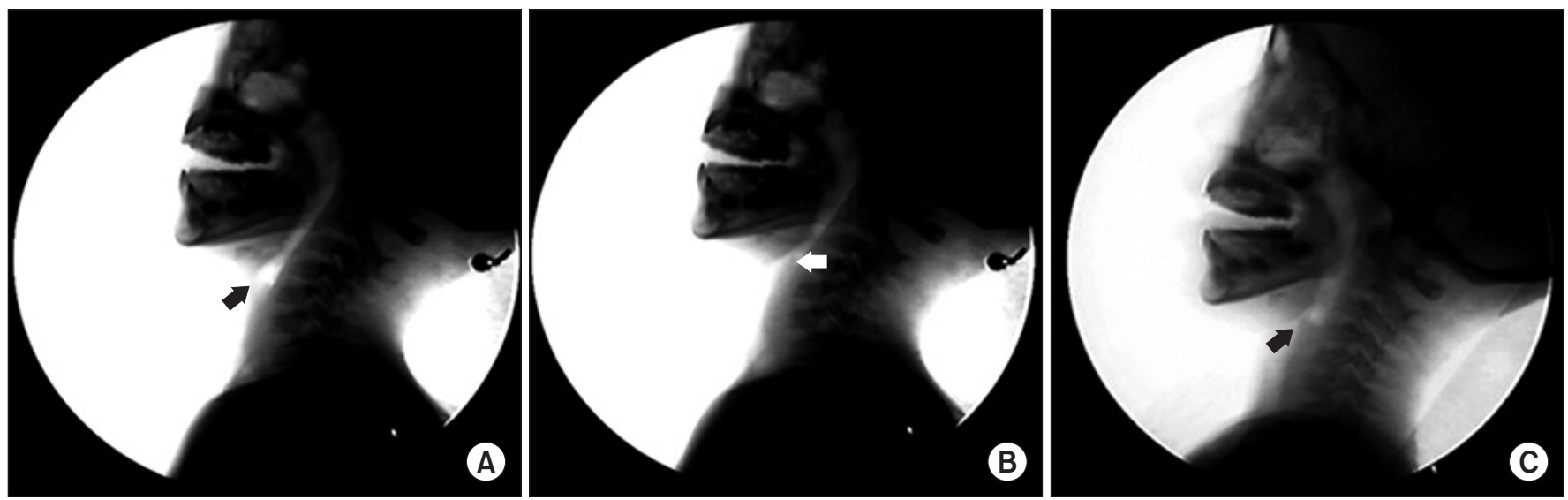

Fig. 2. The arytenoid cartilage in the videofluoroscopic swallowing study (VFSS) shows airway obstruction during inspiration (A) and opening during expiration (B). After 1 month of observation, the anterior dislocation of arytenoid cartilage was alleviated on the VFSS (C).
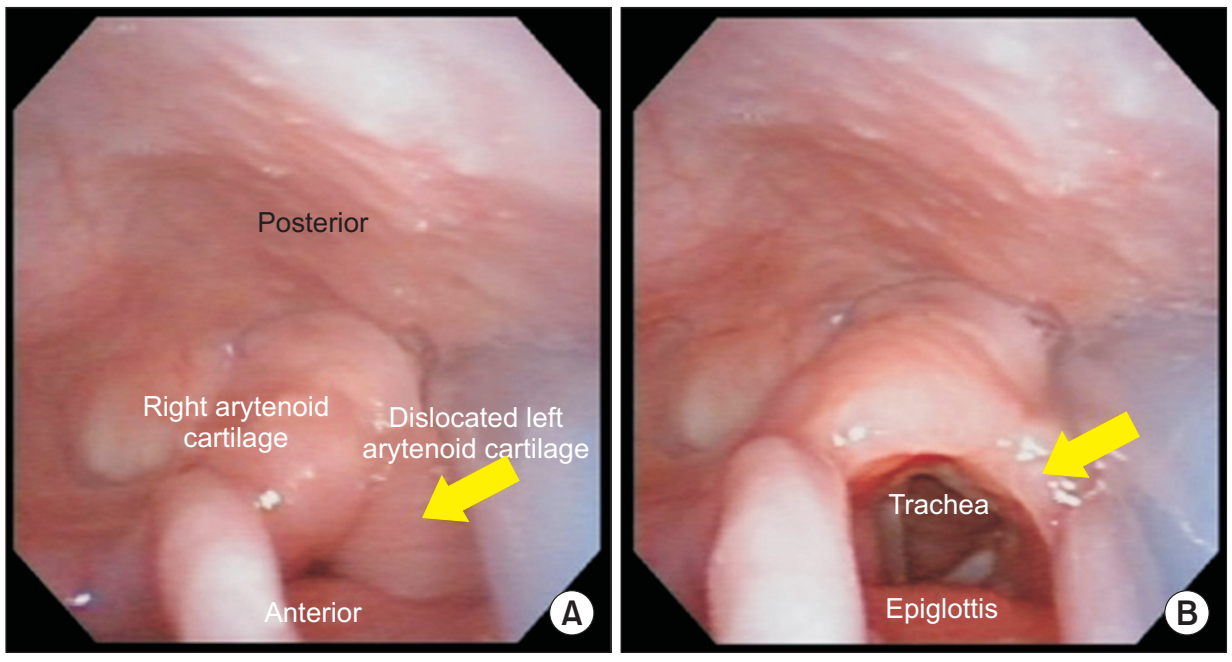

Fig. 3. On the laryngoscope, anterior dislocation of arytenoid cartilage (arrow) during inspiration induces airway obstruction (A) and opening during expiration (B).
VFSS performed after a month (Fig. 2C).

The informed consent was waived.

\section{DISCUSSION}

Dislocation of the arytenoid cartilage is a rare disorder and difficult to diagnose in children. It may be more difficult to diagnose if the patient has cognitive impairment or is unable to speak spontaneously like in this case. Therefore, more accurate and appropriate evaluation is needed for pediatric patients.

Arytenoid cartilage can be dislocated either in the anterior or posterior direction. There are several pathomechanisms associated with dislocation in each direction.
Posterolateral displacement is hypothesized to be caused by the insertion of the distal third of the endotracheal tube when holding the tube with the right hand while holding the laryngoscope with the left hand at the time of intubation, causing the left arytenoid cartilage to subluxate posterolaterally [7]. Additionally, subluxation may occur when extubation is performed without sufficient deflation of the balloon of the endotracheal tube [6]. Conversely, anteromedial displacement of the arytenoid cartilage can occur when the blade of the laryngoscope is inserted to the pyriform sinus and lifted forward, and also when the tip of the endotracheal tube presses the arytenoid cartilage in the forward direction [8]. In our case, the left arytenoid cartilage was detected to have been dis- 
placed to the anteromedial side, possibly due to tracheal intubation, on laryngoscopy.

Dislocation of the arytenoid cartilage in children is rarely reported. Moreover, diagnosis is difficult as symptoms can be subtle, especially with comorbidities causing impaired speech or vocalization, as in this patient. Differential diagnosis of arytenoid cartilage dislocation includes recurrent laryngeal nerve (RLN) injury causing vocal cord immobility. Differentiating RLN injury from arytenoid cartilage dislocation by video laryngoscopy is difficult. For the diagnosis of RLN injury, laryngeal electromyography (EMG) may be helpful; the recruitment pattern of the laryngeal muscle EMG is normal in arytenoid cartilage dislocation, whereas, it is abnormal in RLN injury. Another indicator consistent with arytenoid dislocation is the absence of the "jostle sign", which is a passive medial movement of the paralyzed vocal cord during adduction in cases of vocal cord palsy. In our patient, the jostle sign was not observed during video laryngoscopy, and laryngeal EMG was not required [9].

Prompt diagnosis can help restore vocal function, which may be delayed without prompt and appropriate treatment. Conservative management including voice therapy can be helpful in treating arytenoid cartilage dislocation, and surgical correction of the dislocated arytenoid cartilage should be considered in cases not showing improvement. According to a previous study reporting dislocated arytenoid cartilage in 11 patients, 2 out of 5 patients who refused surgery recovered spontaneously [10]. Although spontaneous reduction is rare, inspiratory stridor improved spontaneously in this case.

We report a case of arytenoid cartilage dislocation incidentally diagnosed during VFSS. Although a CT scan may help in the diagnosis of arytenoid cartilage dislocation, often the cartilage is not ossified in pediatric patients, and hence not detected on a CT scan [1]. Moreover, the position of the arytenoid cartilage may appear normal in a static CT scan if it is taken during expiration. Conversely, the VFSS has an advantage over the CT scan as it enables direct evaluation of dynamic cartilage movement. The VFSS might be useful in the early diagnosis of arytenoid cartilage dislocation in pediatric patients. However, these findings in VFSS are easily missed; therefore, careful evaluation is necessary to help with an accurate assessment of the swallowing function.

\section{CONFLICT OF INTEREST}

No potential conflict of interest relevant to this article was reported.

\section{ACKNOWLEDGMENTS}

This study is supported by a research grant of the Research Institute of Rehabilitation Medicine, Yonsei University College of Medicine for 2017.

\section{AUTHOR CONTRIBUTION}

Conceptualization: Rha DW, Park ES. Methodology: Rha DW, Kim SK. Formal analysis: Lee YH, Park HK. Funding acquisition: Rha DW. Project administration: Rha DW. Visualization: Lee YH, Park JE. Writing - original draft: Lee YH. Writing - review and editing: Rha DW, Park HK, Lee YH. Approval of final manuscript: all authors.

\section{REFERENCES}

1. Rubin AD, Hawkshaw MJ, Moyer CA, Dean CM, Sataloff RT. Arytenoid cartilage dislocation: a 20-year experience. J Voice 2005; 19:687-701.

2. Szigeti CL, Baeuerle JJ, Mongan PD. Arytenoid dislocation with lighted stylet intubation: case report and retrospective review. Anesth Analg 1994;78:185-6.

3. Yamanaka H, Hayashi $Y$, Watanabe $Y$, Uematu $H$, Mashimo T. Prolonged hoarseness and arytenoid cartilage dislocation after tracheal intubation. $\mathrm{Br} \mathrm{J}$ Anaesth 2009;103:452-5.

4. Close LG, Merkel M, Watson B, Schaefer SD. Cricoarytenoid subluxation, computed tomography, and electromyography findings. Head Neck Surg 1987;9:341-8.

5. Yin SS, Qiu WW, Stucker FJ. Value of electromyography in differential diagnosis of laryngeal joint injuries after intubation. Ann Otol Rhinol Laryngol 1996;105:446-51.

6. Dudley JP, Mancuso AA, Fonkalsrud EW. Arytenoid dislocation and computed tomography. Arch Otolaryngol 1984;110:483-4.

7. Quick CA, Merwin GE. Arytenoid dislocation. Arch Otolaryngol 1978;104:267-70.

8. Niwa Y, Nakae A, Ogawa M, Takashina M, Hagihira S, Ueyama $\mathrm{H}$, et al. Arytenoid dislocation after cardiac 
surgery. Acta Anaesthesiol Scand 2007;51:1397-400.

9. Goz V, Qureshi S, Hecht AC. Arytenoid dislocation as a cause of prolonged hoarseness after cervical discectomy and fusion. Global Spine J 2013;3:47-50.
10. Mallon AS, Portnoy JE, Landrum T, Sataloff RT. Pediatric arytenoid dislocation: diagnosis and treatment. J Voice 2014;28:115-22. 